\title{
Performance Degradation Assessment of Rolling Element Bearings using Improved Fuzzy Entropy
}

\author{
Keheng Zhu' ${ }^{1}$, Xiaohui Jiang ${ }^{1}$, Liang $\mathrm{Chen}^{2}$, Haolin $\mathrm{Li}^{1}$ \\ ${ }^{1}$ School of Mechanical Engineering, University of Shanghai for Science and Technology, Jungong Road, Shanghai 200093, \\ PRChina, khzhdl@gmail.com \\ ${ }^{2}$ School of Energy and Power Engineering, Dalian University of Technology, Linggong Road, Dalian 116023, PR China
}

\begin{abstract}
Rolling element bearings are an important unit in the rotating machines, and their performance degradation assessment is the basis of condition-based maintenance. Targeting the non-linear dynamic characteristics of faulty signals of rolling element bearings, a bearing performance degradation assessment approach based on improved fuzzy entropy (FuzzyEn) is proposed in this paper. FuzzyEn has less dependence on data length and achieves more freedom of parameter selection and more robustness to noise. However, it neglects the global trend of the signal when calculating similarity degree of two vectors, and thus cannot reflect the running state of the rolling element bearings accurately. Based on this consideration, the algorithm of FuzzyEn is improved in this paper and the improved FuzzyEn is utilized as an indicator for bearing performance degradation evaluation. The vibration data from run-to-failure test of rolling element bearings are used to validate the proposed method. The experimental results demonstrate that, compared with the traditional kurtosis and root mean square, the proposed method can detect the incipient fault in advance and can reflect the whole performance degradation process more clearly.
\end{abstract}

Keywords: Performance degradation assessment, improved fuzzy entropy, rolling element bearing.

\section{INTRODUCTION}

Machinery condition monitoring has received considerable attention for many years because a reliable condition monitoring system can significantly reduce the huge cost due to the unplanned downtime [1]-[2]. Rolling element bearings are the most widely used component in the rotating machines, and they are also one of the most easily damaged mechanical parts. The running state of bearing influences the reliable operation of the whole machine. Therefore, the bearing condition monitoring is of great importance to keep machines running reliably and reduce economical loss. Among various condition monitoring methods, the most suitable and effective one is vibration analysis because vibration signals of rolling element bearings include abundant fault information [3]-[4].

Diagnosis and prognosis are the two important aspects of condition-based maintenance. Performance degradation assessment is the base of prognosis which is much more efficient than fault diagnosis to achieve zero-downtime performance [1]. It requires reflecting the comprehensive performance degradation degree based on the extracted features and focuses on the vibration trend over a whole lifetime while traditional fault diagnosis only needs to be capable of identifying different types of faults. To date, many researches on the performance degradation assessment of rolling element bearings have been carried out, most of which are based on the intelligent assessment approaches, such as self-organizing map (SOM) [5]-[6], hidden Markov model (HMM) [7], support vector data description (SVDD) [8]-[10], Gaussian mixture model (GMM) [11] and logistic regression (LR) [12]. However, these proposed intelligent assessment models may all exhibit their own limitations [13]-[14]. For example, ANN-based model operates as a "black box" without documentation of qualitative information of the model and requires large amount of training data [13], HMM-based model needs assumptions which are unpractical in the real applications [14] and Gaussian process-based model is only suitable for Gaussian likelihood and has a heavy computation burden [13].

On the other hand, designing proper indicators without intelligent models has not received enough attention in previous studies, and some existing statistical indicators in the time domain have their own advantages and limitations as well. For example, the extensively used root mean square (RMS) can steadily grow with the defect evolution of the bearing, but it is insensitive to the early faults. On the contrary, kurtosis and crest factor are sensitive enough to impulse defect at the incipient stage but reduce to normallike levels as the damage grows [7]. Hence, exploring new effective indicators, which can reflect the comprehensive degeneration process of rolling element bearings, is valuable work [15]. 
As it is known, vibration signals of a bearing, especially when fault occurs, will present non-linear characteristics due to the non-linear factors such as stiffness, friction and clearance [16]. For this reason, the traditional time and frequency domain signal analysis methods based on the linear systems, even the advanced signal processing methods such as wavelet transform, are usually hard to make an accurate assessment of the bearing running states. With the development of nonlinear dynamics theory, various nonlinear analysis approaches have offered a good alternative to identify and predict the complicated non-linear dynamic behavior of bearings, among which entropy-based parameters, such as appropriate entropy (ApEn), sample entropy (SampEn), multi-scale entropy (MSE), and hierarchical entropy (HE) have been researched and introduced to the field of bearing fault diagnosis [17]-[19]. Due to the bias caused by self-matching, ApEn depends heavily on the data length and its estimated value is uniformly lower than the expected one, especially as short data concerned, and lacks relative coherence as well [20]. In order to overcome the shortcomings of ApEn, Richman and Moorman [21] developed another related measure of signal complexity, viz. SampEn, which is less dependent on dataset length and has better relative consistency. However, the definitions of similarity between vectors in SampEn and ApEn are both based on Heaviside function, whose boundary is discontinuous and rigid. Due to the inherent flaws of Heaviside function, there still exist problems in the validity of the entropy definition. In order to address the problems, Chen et al. [22] presented recently a measure of complexity named fuzzy entropy (FuzzyEn) by improving the original SampEn. In comparison with SampEn, FuzzyEn uses fuzzy membership functions to replace Heaviside function for making a soft and continuous boundary, which makes FuzzyEn well defined at small parameters and enables it to change continuously. Nevertheless, the fuzzy function used in FuzzyEn by Chen et al. was deemed to lack clear physical meanings [23]. Therefore, Zheng et al. [24] defined a new fuzzy function and used the optimized one to measure the complexity of vibration signals of rolling element bearings. However, the limitation of FuzzyEn is that it neglects the global characteristics of the signal and thus may produce inaccurate results [25].

As a bearing runs from a normal condition to a faulty one, the vibration signals will present different characteristics with the bearing state varying. Moreover, the fault severity and growth of rolling element bearings have been proved by the previous researches that they are associated with the changes of the entropy values (such as ApEn, MSE and HE), which makes them attractive tools for monitoring system dynamics [17]-[19]. In this study, inspired by these existing researches, we modified the algorithm of FuzzyEn to relieve the aforementioned limitation and used the improved FuzzyEn as an indicator for bearing performance degradation. Considering the fact that the global characteristics and global trends of bearing vibration signals may change as the bearing degenerates gradually, the improved FuzzyEn should be more suitable to reflect the bearing degradation process over its whole lifetime. The effectiveness of the modified FuzzyEn for bearing performance degradation evaluation is then investigated through experimental data.

The paper is organized as follows. Section 2 is dedicated to the improved FuzzyEn method. The vibration data from a laboratory test-to-failure test are employed to validate the effectiveness of the proposed method in section 3. In section 4 , conclusions from this research are presented.

\section{THE IMPROVED FUZZY ENTROPY METHOD}

\subsection{Fuzzy entropy}

SampEn is a refinement of ApEn and is free of bias caused by self-matching. Details on the calculation of SampEn can be found in previous researches [21]-[23]. The computation of the vectors' similarity in SampEn is defined based on Heaviside function, which is discontinuous and makes a binary decision on the similarity between vectors according to their distance. However, in the real physical world, it is hard to decide whether a pattern belongs to a given class because boundaries between classes may be ambiguous. Hence, FuzzyEn is developed to overcome the drawback of SampEn by replacing the Heaviside function with a continuous Gaussian type function, which is utilized to measure the similarity degree instead of a binary decision.

The FuzzyEn algorithm can be described as follows [22]:

1. For a time series of $N$ points $\{u(i): 1 \leq i \leq N\}$, form the $m$ length vectors,

$$
\begin{array}{r}
X_{i}^{m}=\{u(i), u(i+1), \ldots, u(i+m-1)\} \\
-u_{0}(i)
\end{array}
$$

where $1 \leq i \leq N-m+1$ and $X_{i}^{m}$ denotes $m$ consecutive $u$ values, commencing with the $i$ th point and generalized by removing their baseline

$$
u_{0}(i)=m^{-1} \sum_{j=0}^{m-1} u(i+j)
$$

2. The distance between $X_{i}^{m}$ and $X_{j}^{m}$ is defined as the maximum absolute difference of the corresponding scalar components

$$
\begin{aligned}
d_{i j}^{m}= & d\left[X_{i}^{m}-X_{j}^{m}\right] \\
=\max _{k \in[0, m-1]} \mid & \left(u(i+k)-u_{0}(i)\right)- \\
& \left(u(j+k)-u_{0}(j)\right) \mid
\end{aligned}
$$

3. The similarity degree $D_{i j}^{m}$ of $X_{i}^{m}$ to $X_{j}^{m}$ is calculated with a fuzzy function

$$
D_{i j}^{m}=\mu\left(d_{i j}^{m}, r\right)
$$


4. For each $X_{i}^{m}$, average all the similarity degrees of its neighboring vectors $X_{j}^{m}$, then designate

$$
\varphi_{i}^{m}(r)=(N-m-1)^{-1} \sum_{j=1, j \neq i}^{N-m} D_{i j}^{m}
$$

5. Define the function $\varphi^{m}(r)$ as

$$
\varphi^{m}(r)=(N-m)^{-1} \sum_{i=1}^{N-m} \varphi_{i}^{m}(r)
$$

6. Similarly, for the dimension $m+1$, repeat the previous procedures and the $\varphi^{m+1}(r)$ is defined as

$$
\varphi^{m+1}(r)=(N-m)^{-1} \sum_{i=1}^{N-m} \varphi_{i}^{m+1}(r)
$$

7. FuzzyEn of the time series is then denoted as

$$
\begin{aligned}
& \operatorname{FuzzyEn}(m, r) \\
& =\lim _{N \rightarrow \infty}\left[\ln \varphi^{m}(r)-\ln \varphi^{m+1}(r)\right]
\end{aligned}
$$

8. Finally, for a finite number of data points $N$, its estimation can be estimated by the statistic

$$
\begin{aligned}
& F u z z y \operatorname{En}(m, r, N) \\
& =\ln \varphi^{m}(r)-\ln \varphi^{m+1}(r)
\end{aligned}
$$

Originally, the exponential function was adopted to fuzzily measure the similarity between vectors by Chen et al. [22], which was defined as

$$
\mu\left(d_{i j}^{m}, r, n\right)=e^{-\left(d_{i j}^{m} / r\right)^{n}}
$$

In order to make FuzzyEn more physically meaningful, Zheng et al. [24] designed a new membership function to substitute the original one as

$$
\mu\left(d_{i j}^{m}, r, n\right)=e^{-\ln 2\left(d_{i j}^{m} / r\right)^{n}}
$$

In this paper, equation (11) is applied to compute FuzzyEn of bearing vibration signals, and an assessment of its performance is made.

\subsection{The improved fuzzy entropy}

A change in the definition of the aforementioned FuzzyEn is the formation of the vector $X_{i}^{m}$, which is generalized by removing a baseline defined by (2). However, this implementation of removing the baseline focuses only on the local characteristics of the signal and neglects its global trend [25]. As for the bearing vibration signals, their global characteristics may vary with the running states of bearings deteriorating gradually. Therefore, it may be not enough to reflect the bearing performance degradation process effectively by using the original FuzzyEn. Based on this consideration, we modified (1) as

$$
X_{i}^{m}=\{u(i), u(i+1), \ldots, u(i+m-1)\}
$$

Which means the local mean is removed. In this study, in order to reflect the bearing degradation states more accurately, equation (12) was utilized to calculate the values of FuzzyEn. The modified FuzzyEn is then employed to measure the complexity of vibration signals of rolling element bearings, and the obtained entropy value is defined as the bearing degradation indicator. Finally, the performance of the improved FuzzyEn for bearing degradation evaluation was assessed with experimental data.

\subsection{Parameters selection}

There are four parameters that need to be selected for each calculation of FuzzyEn, which is $m, r, N$, and $n$, respectively. The parameter $\mathrm{m}$ is the length of sequences to be compared. Typically, larger $\mathrm{m}$ allows more detailed reconstruction of the dynamic process [22]. However, a too large $m$ value needs a very large $N\left(10^{m}-30^{m}\right)$, which is unfavorable in many real applications. Generally, $m$ is chosen as 2 , and $N$ is selected as 2048 in this paper. The width and gradient of the boundary of the exponential function are determined by the parameters $r$ and $n$, respectively, viz. the fuzzy similarity boundary. A too narrow boundary will result in noticeable influence from noise while a too broad one will lead to the loss of information. According to the previous studies [18][19], $r=0.1-0.25 \mathrm{SD}$ (standard deviation of the original data) and $n$ should be small integers. To seek a balance between noise robustness and information loss, $r=0.2 \mathrm{SD}$ is assigned, and $n=2$ is selected.

\section{EXPERIMENTAL VALIDATION}

\subsection{Experimental data}

In order to investigate the effectiveness of the modified FuzzyEn for the rolling element bearing performance degradation assessment, vibration signals from run-tofailure experiments are analyzed. The bearing vibration data is from the prognostic data repository contributed by the Center on Intelligent Maintenance Systems (IMS), University of Cincinnati [26].

The bearing test rig is shown in Fig.1., which consists of one shaft, four test bearings, an $\mathrm{AC}$ motor and rub belts. Four Rexnord ZA-2115 double row bearings were installed on one shaft. The shaft was driven by the AC motor and coupled by rub belts. The rotational speed was kept constantly at $2000 \mathrm{rpm}$ and a radial load of $6000 \mathrm{lbs}$ was applied to the shaft and bearing through a spring mechanism. 
The lubrication of all the bearings was provided by an oil circulation system and the flow and temperature of the lubricant was regulated by the system. A magnetic plug was installed in the oil feedback pipe to collects debris from the oil as evidence of bearing degradation. When the accumulated debris adhering to the magnetic plug exceeded a certain level, an electrical switch closed and the test stopped. A PCB 352B33 High Sensitivity Quarts ICP accelerometer was installed on each bearing housing to collect the vibration signals. The vibration data was collected every $10 \mathrm{~min}$ at a sampling frequency of $20 \mathrm{kHz}$ and the data length was 20480 points.

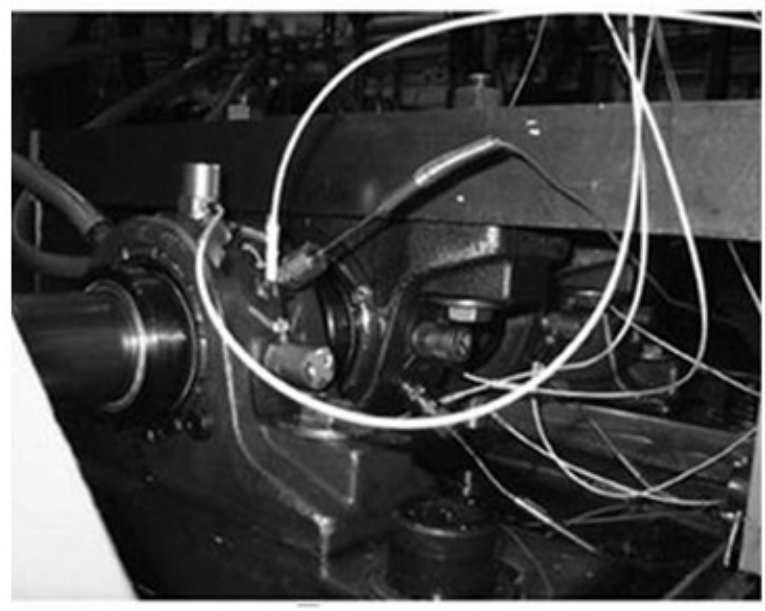

Fig.1. Bearing test rig.

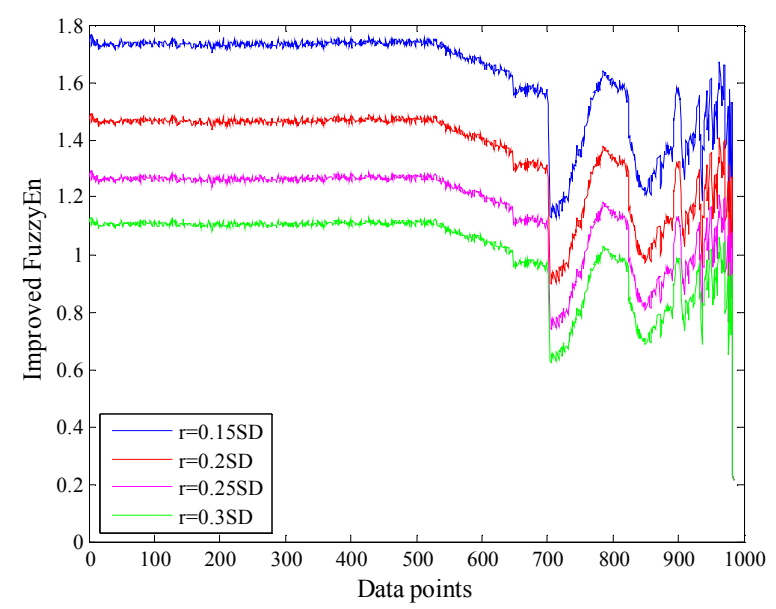

Fig.2. Effect of parameter $r$ on the improved FuzzyEn values.

\subsection{Effects of parameter selection}

As mentioned above, the calculation of FuzzyEn depends less on dataset length $N$ and a too large $m$ value requires a very large $N$, which is unpractical in many real applications. In the present study, based on the aforementioned experimental signals, the effects of parameters $r$ and $n$ on the computation of the improved FuzzyEn have been investigated. The effect of different $r(n=2)$ on the FuzzyEn values is shown in Fig.2., from which it can be seen that the larger the $r$ is, the smaller the entropy value is. Although they vary with $r$ increasing, the FuzzyEn values keep the same variation trend as the $r$ changed. The effect of different $n(r=0.2 \mathrm{SD})$ on the FuzzyEn values is given in Fig.3. From Fig.3., we can see that the larger the $n$ is, the larger the entropy value is. Nevertheless, similarly to $r$, the FuzzyEn values keep the same variation trend with $n$ changing. From Fig.2. and Fig.3., it can be observed that the changes of parameter $r$ and $n$ have little influence on the bearing performance degradation assessment. Considering all the above factors, in this paper, $m$ is fixed as 2 , and $N$ is chosen as 2048 while $r=0.2 \mathrm{SD}$ is selected, and $n=2$ is assigned.

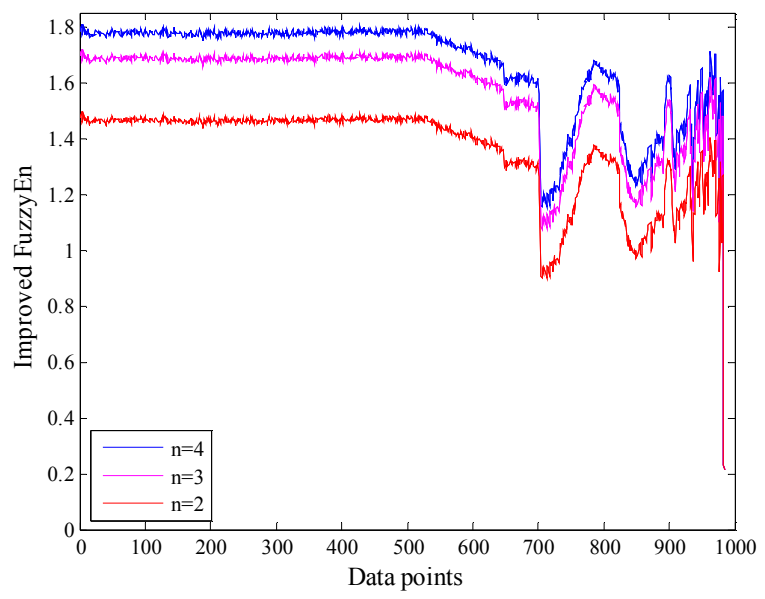

Fig.3. Effect of parameter $n$ on the improved FuzzyEn values.

\subsection{Results and analysis}

The bearing vibration signals from test 2 are employed for analysis. At the end of this experiment, an outer race defect was found in bearing 1 . There are 984 data files being collected in this experiment. The FuzzyEn values of the bearing vibration data are then calculated and used as the bearing degradation indicator. In order to avoid the influence of random factors on the computation of FuzzyEn values, each data file is separated into 10 segments (each segment length is 2048), and the mean value of FuzzyEn of these 10 segments can be obtained.

The variations of SampEn, FuzzyEn and improved FuzzyEn with time are shown in Fig.4., respectively. It can be seen that the entropy values of SampEn and improved FuzzyEn show a similar trend while the values of original FuzzyEn present a different way. The variance trends of SampEn and improved FuzzyEn demonstrate that the entropy values keep stable for a long-time period when bearing is running under normal condition and begin to decrease as early fault occurs, which means that the vibration signals of healthy bearing are more complex than those of faulty bearing. According to the literature [24], this can be explained by the fact that the vibration signals under normal condition have lower self-similarity because of its randomness and irregularity, and the self-similarity will increase as the fault appears. As for the original FuzzyEn, some of its values in bearing degradation area (nearby the 
final failure) are much larger than these under healthy condition, which will result in mistaken decision. This may be due to the fact that it subtracts the local mean during the algorithm implementation and thus neglects the global trend of bearing vibration signal. In addition, compared with SampEn, there exists a smaller range of fluctuation in the improved FuzzyEn values during the bearing's whole lifetime, which means improved FuzzyEn presents a better statistical stability than SampEn. This is because a fuzzy membership function is adopted in improved FuzzyEn to define the decision rule for vector similarity while a piecewise function is used in SampEn. Therefore, the improved FuzzyEn is most suitable for bearing degradation assessment.

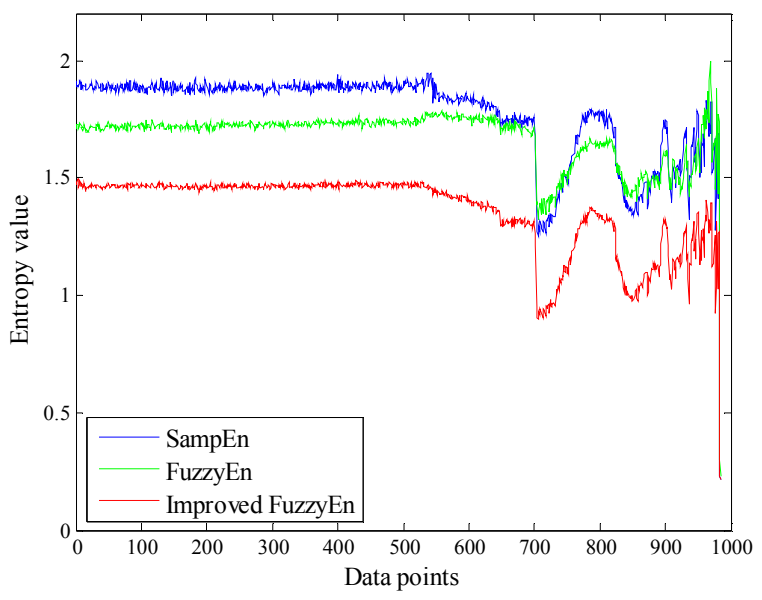

Fig.4. Comparison of SampEn, FuzzyEn and improved FuzzyEn over bearing's whole lifetime.

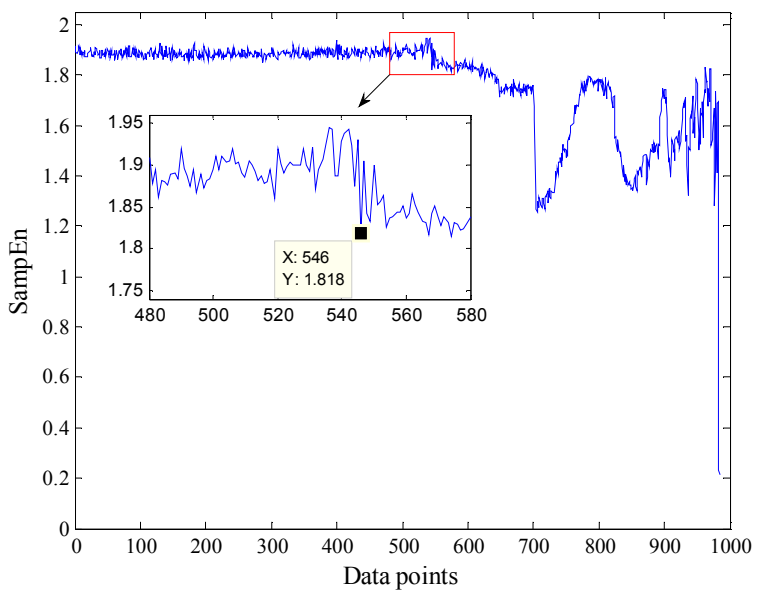

Fig.5. The variation of SampEn values with time.

In order to further show the advantage of the improved FuzzyEn over SampEn, the corresponding curves with local enlargement are illustrated in Fig.5. and Fig.6., respectively. From Fig.5. and Fig.6., it can be observed that the SampEn values indicate the occurrence of initial degradation at the 546th data point while the improved FuzzyEn values start to decrease at the 533th data point, which means that the improved FuzzyEn can detect the incipient degradation 13 data points in advance. For comparison purpose, another two degradation assessment indicators, root mean square (RMS) and the kurtosis value, are also calculated and shown in Fig.7. and Fig.8., respectively. It can be seen from Fig.7. that RMS values indicate the occurrence of degradation at the 535th data point, which is two data points behind compared with the values of improved FuzzyEn. Moreover, the increase of RMS values is less obvious than that of improved FuzzyEn. From Fig.8., it can be observed that the bearing performance degradation begins at about the 648th data point, which is more than 100 data points later than that indicated by improved FuzzyEn. In addition, the kurtosis values decrease sharply as the occurrence of final failure, which makes the prediction of bearing degradation degree impossible.

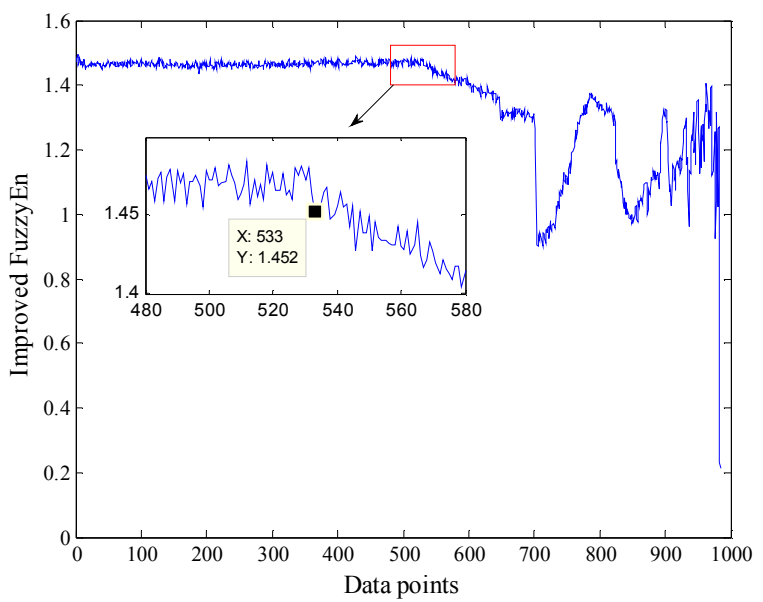

Fig.6. The variation of values of improved FuzzyEn with time.

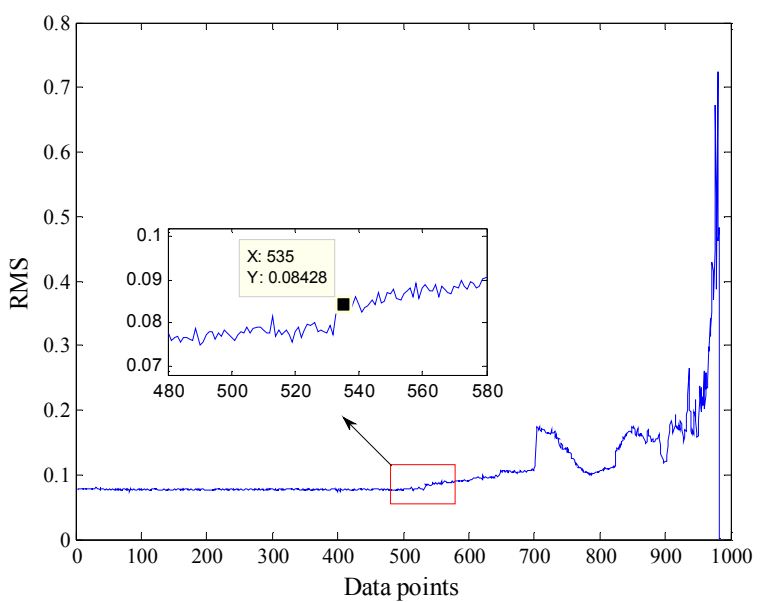

Fig.7. The variation of RMS values with time.

According to the above analysis, the following can be observed: (1) Compared with SampEn and original FuzzyEn, the improved FuzzyEn is the most suitable for performance degradation evaluation of rolling element bearings. (2) The modified FuzzyEn values can assess the bearing 
performance degradation process over their whole life time clearly and effectively. (3) In contrast with kurtosis and RMS, the modified FuzzyEn can detect the bearing initial degradation in advance, which is of great significance for the prognosis in the condition monitoring.

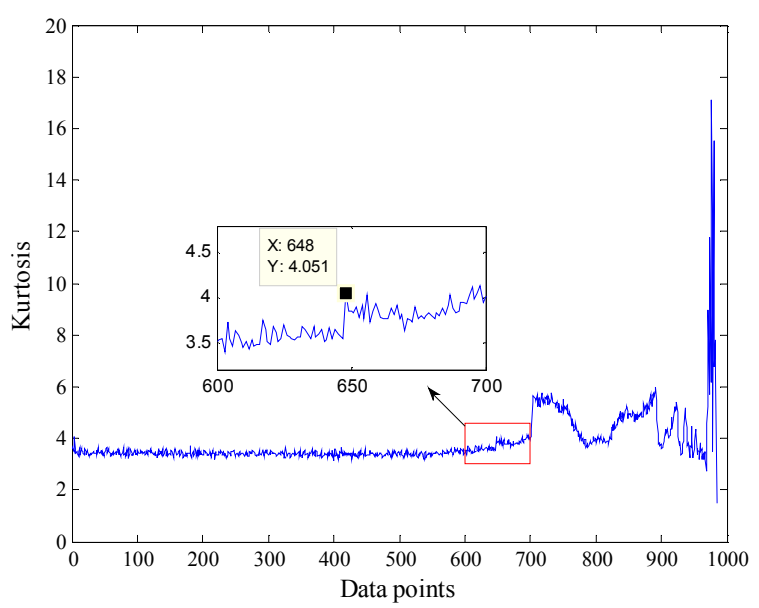

Fig.8. The variation of kurtosis values with time.

\section{CONCLUSIONS}

In this paper, the FuzzyEn is improved and used as an indicator for the performance degradation assessment of rolling element bearings. The run-to-failure vibrational signals are applied to verify the suitability of the improved FuzzyEn method. The experimental results demonstrate that the modified FuzzyEn can reflect the bearing degradation process effectively and has shown better performance than SampEn and original FuzzyEn. Compared with RMS, the improved FuzzyEn can indicate the initial degradation two data points in advance and presents a more obvious increase. In contrast with kurtosis, the improved FuzzyEn can detect the occurrence of degradation more than 100 data points ahead and shows a clearer and more accurate trend for bearing degradation process over its whole lifetime.

\section{ACKNOWLEDGMENT}

This work was supported by the National Natural Science Foundation of China (Grant No. 51505291). The authors are grateful to the Center for Intelligent Maintenance System, University of Cincinnati, USA, for providing the experimental data.

\section{REFERENCES}

[1] Jardine, A.K.S., Lin, D.M., Banjevic, D. (2006). A review on machinery diagnostics and prognostics implementing condition-based maintenance. Mechanical Systems Signal Processing, 20 (7), 14831510.

[2] Huang, H.F., Ouyang, H.J., Gao, H.L., Guo, L., Li, D., Wen, J. (2016). A feature extraction method for vibration signal of bearing incipient degradation. Measurement Science Review, 16 (3), 149-159.
[3] Tandon, N., Choudhury, A. (1999). A review of vibration and acoustic measurement methods for the detection of defects in rolling element bearings. Tribology International, 32 (8), 469-480.

[4] Gebraeel, N., Lawley, M., Liu, R., Parmeshwaran, V. (2004). Residual life predictions from vibration-based degradation signals: A Neural Network approach. IEEE Transactions on Industrial Electronics, 51 (3), 694-700.

[5] Qiu, H., Lee, J., Lin, J., Yu, G. (2003). Robust performance degradation assessment methods for enhanced rolling element bearing prognostics. Advanced Engineering Informatics, 17 (3), 127-140.

[6] Huang, R.Q., Xi, L.F., Li, X.L., Liu, C.R., Qiu, H., Lee, J. (2007). Residual life predictions for ball bearings based on self-organizing map and back propagation neural network methods. Mechanical Systems Signal Processing, 21 (1), 193-207.

[7] Ocak, H., Loparo, K.A., Discenzo, F.M. (2007). Online tracking of bearing wear using wavelet packet decomposition and probabilistic modeling: A method for bearing prognostics. Journal of Sound Vibration, 302 (4), 951-961.

[8] Pan, Y.N., Chen, J., Guo, L. (2009). Robust bearing performance degradation assessment method based on improved wavelet packet-support vector data description. Mechanical Systems Signal Processing, 23 (3), 669-681.

[9] Shen, Z.J., He, Z.J., Chen, X.F., Sun, C., Liu, Z. (2012). A monotonic degradation assessment index of rolling bearings using fuzzy support vector data description and running time. Sensors, 12 (8), 1010910135.

[10] Zhu, X.R., Zhang, Y.Y., Zhu, Y.S. (2013). Bearing performance degradation assessment based on the rough support vector data description. Mechanical Systems Signal Processing, 34 (1), 203-217.

[11] $\mathrm{Yu}$, J.B. (2011). Bearing performance degradation assessment using locality preserving projections and Gaussian mixture models. Mechanical Systems Signal Processing, 25 (7), 2573-2588.

[12] Caesarendra, W., Widodo, A., Thom, P.H., Yang, B.S., Setiawan, J.D. (2011). Combine probability approach and indirect data-driven method for bearing degradation prognostics. IEEE Transactions Reliability, 60 (1), 14-20.

[13] Kan, M.S., Tan, A.C.C., Mathew, J. (2015). A review on prognostic techniques for non-stationary and nonlinear rotating systems. Mechanical Systems Signal Processing, 62-63, 1-20.

[14] Peng, Y., Dong, M., Zuo, M.J. (2010). Current status of machine prognostics in condition-based maintenance: A review. International Journal of Advance Manufacturing Technology, 50 (1), 297-313

[15] Dong, G.M., Chen, J. (2010). Study on cyclic energy indicator for degradation assessment of rolling element bearings. Journal of Vibration and Control, 17 (12), 1805-1816. 
[16] Yang, Y., Yu, D.J., Cheng, J.S. (2006). A roller bearing fault diagnosis method based on EMD energy entropy and ANN. Journal of Sound and Vibration, 294 (1), 269-277.

[17] Yan, R.Q., Gao, R.X. (2007). Approximate entropy as a diagnostic tool for machine health monitoring. Mechanical Systems Signal Processing, 21 (2), 824839.

[18] Zhang, L., Xiong, G.L., Liu, H.S., Zou, H., Guo, W. (2010). Bearing fault diagnosis using multi-scale entropy and adaptive neuro-fuzzy inference. Expert Systems with Applications, 37 (8), 6017-6085.

[19] Zhu, K.H., Song, X.G., Xue, D.X. (2014). A roller bearing fault diagnosis method based on hierarchical entropy and support vector machine with particle swarm optimization algorithm. Measurement, 47, 669675.

[20] Pincus, S.M. (1991). Approximate entropy as a measure of system complexity. Proceedings of the National Academy of Sciences, 88 (6), 2297-2301.

[21] Richman, J.S., Moorman, J.R. (2000). Physiological time-series analysis using approximate entropy and sample entropy. American Journal of PhysiologyHeart and Circulatory Physiology, 278 (6), H2039H2049.
[22] Chen, W.T., Wang, Z.Z., Xie, H.B., Yu, W. (2007). Characterization of surface EMG signal based on fuzzy entropy. IEEE Transactions on Neural Systems and Rehabilitation Engineering, 15 (2), 266-272.

[23] Xiong, G.L., Zhang, L., Liu, H.S., Zou, H.J., Guo, W.Z. (2010). A comparative study on ApEn, SampEn and their fuzzy counterparts in a multiscale framework for feature extraction. Journal of Zhejiang University SCIENCE A, 11 (4), 270-279.

[24] Zheng, J.D., Cheng, J.S., Yang, Y., Luo, S. (2014). A rolling bearing fault diagnosis method based on multiscale fuzzy entropy and variable predictive modelbased class discrimination. Mechanism and Machine Theory, 78, 187-200.

[25] Liu, C.Y., Li, K., Zhao, L.N., Liu, F., Zheng, D., Liu, C., Liu, S. (2013). Analysis of heart rate variability using fuzzy measure entropy. Computers in Biology and Medicine, 43 (2), 100-108

[26] Lee, J., Qiu, H., Yu, G., Lin, J. (2007). “Bearing Data Set”, NASA Ames Prognostics Data Repository. http://ti.arc.nasa.gov/tech/dash/pcoe/prognostic-datarepository, IMS, University of Cincinnati, Rexnord Technical Services. (Accessed 13 April 2014)

Received April 10, 2017. Accepted September 20, 2017. 